\title{
Salidroside protects against ox-LDL-induced endothelial injury by enhancing autophagy mediated by SIRT1-FoxO1 pathway
}

\author{
Zhongsheng Zhu* ${ }^{*}$, Jinyu Li and Xiaorong Zhang
}

\begin{abstract}
Background: Atherosclerosis is a condition with the vascular accumulation of lipid plaques, and its main major contributing factor is endothelial injury induced by oxidized low-density lipoprotein (ox-LDL). Salidroside (SAL) is the primary active ingredient of Rhodiola rosea, and exhibits antioxidant properties on endothelial cells and alleviates atherosclerosis. However, the effect of SAL on autophagy in ox-LDL-induced vascular endothelial injury remains unclear. Here, we investigated the effect and underlying mechanisms of SAL on autophagy in human umbilical vein endothelial cells (HUVECs).
\end{abstract}

Methods: HUVECs were incubated with ox-LDL to induce in vitro atherosclerosis model. The cell viability and injury were evaluated by cell counting kit-8 (CCK-8) assay and lactate dehydrogenase (LDH) release assay. The oxidative stress was evaluated by NADPH oxidase, malondialdehyde (MDA) and superoxide dismutase (SOD) activities. Immunofluorescence was performed to detect autophagy using LC3 $\beta$ antibody. Quantitative real-time PCR (qRT-PCR) and western blot were performed to measure the mRNA expressions of SIRT1 and Forkhead box 01 (FOXO1). Nicotinamide (NAM) and AS1842856 were used to inhibit activities of SIRT1 and FOXO1, respectively.

Results: Exposure of HUVECs to ox-LDL $(100 \mu \mathrm{g} / \mathrm{mL})$ reduced cell viability, increased cellular MDA, and reduced SOD in a concentration-dependent manner. The pretreatment with SAL (20, 50 and $100 \mu \mathrm{M})$ significantly enhanced the cell viability and decreased LDH release in HUVECs exposed to ox-LDL $(100 \mu \mathrm{g} / \mathrm{mL})$. ox-LDL induced autophagy in HUVECs, which was further enhanced by pretreatment with SAL. However, SAL attenuated increase in oxidative stress in HUVECs induced by Ox-LDL. Ox-LDL reduced mRNA and protein expressions of SIRT1 and FOXO1, which could be reversed by SAL. The protective, anti-oxidative and pro-autophagic effects of SAL could be obviously abolished by cotreatment with SIRT1 inhibitor or FOXO1 inhibitor.

Conclusion: Salidroside shows protective effect on endothelial cell induced by ox-LDL, and the mechanisms might be related to autophagy induction via increasing SIRT1 and FoxO1 expressions.

Keywords: Atherosclerosis, Salidroside (SAL), Oxidized low-density lipoprotein (ox-LDL), Endothelial cell, Oxidative stress, Autophagy

\footnotetext{
*Correspondence: zhuzssh@163.com

Department of Cardiology, Shanghai Pudong Hospital, Fudan University

Pudong Medical Center, No.2800 Gongwei Road, Pudong New District,

Shanghai 201399, China
}

(c) The Author(s). 2019 Open Access This article is distributed under the terms of the Creative Commons Attribution 4.0 International License (http://creativecommons.org/licenses/by/4.0/), which permits unrestricted use, distribution, and reproduction in any medium, provided you give appropriate credit to the original author(s) and the source, provide a link to the Creative Commons license, and indicate if changes were made. The Creative Commons Public Domain Dedication waiver (http://creativecommons.org/publicdomain/zero/1.0/) applies to the data made available in this article, unless otherwise stated. 


\section{Background}

Atherosclerosis is characterized by accumulation of lipid plaques in vascular endothelium [1]. Endothelial injury is initial event and contributing factor of atherosclerosis, and is mainly caused by oxidized low-density lipoprotein (ox-LDL) [2]. ox-LDL destroys the oxidation-reduction equilibrium of vascular endothelial cells and induces apoptosis of endothelial cells, thus contributing to endothelial injury [3]. Oxidative stress promotes low density lipoprotein (LDL) oxidation of the vascular wall by increasing the superoxide anion, and large amount of ox-LDL further damages vascular endothelium [4]. Endothelial injury is the initial but reversible step in the development of atherosclerosis [5]. Therefore, prevention of endothelial injury has become an promising therapeutic strategy for reversing atherosclerosis.

Autophagy is a highly regulated metabolic process in which long lived proteins and organelles are degraded through the lysosomal system in unfavorable environment. Autophagy is involved in variety of physiological and pathological conditions, including oxidative stress, inflammation, starvation and immune responses [6]. Autophagy plays essential roles in homeostasis and function of heart and vessel, and defective or excessive autophagy leads to atherosclerosis and other cardiovascular disorders [7]. In fact, autophagy shows both protective and aggravating effects on vascular injury in atherosclerosis. Autophagy participates in the defense mechanism against oxidative stress, thereby preventing vascular cell apoptosis [8]. Autophagy also destroys most cytosols and organelles, ultimately leading to endothelial cell death (autophagy death) and plaque instability [9]. Therefore, the precise role of autophagy in the treatment of atherosclerosis by various agents should be investigated in different in vitro systems and animal models.

SIRT1 is a member of the NAD +-dependent deacetylases, and SIRT1 deficiency in endothelial cells promotes oxidative stress, inflammation, foam cell formation, and increased progression of atherosclerosis [10]. SIRT1 is also a promotor of autophagy and SIRT1 inhibition accelerate atherosclerotic plaque development through impaired autophagy in ApoE (-/-) mice [11]. Forkhead box O1 (FOXO1) is a transcription factor and involves a series of intracellular functions, including autophagy, mitochondrial dysfunction and apoptosis [12]. FOXO1 is a potent inhibitor of oxidative stress and thus considered as a therapeutic target for diseases with excessive oxidative stress [13]. FOXO1 is strongly expressed in atherosclerotic plaques and shows atheroprotective effect, as FOXO1 silencing in endothelial cells prevented atherosclerosis in mouse model [14]. Until now, the roles of SIRT1 and FOXO1 in atherosclerosis as therapeutic target remain largely unknown.
Salidroside (SAL) is the main ingredient of Rhodiola rosea, with suppressive effects on oxidative stress [15]. Salidroside protected foam cells against injury induced by ox-LDL, and alleviated atherosclerosis in apoE(-/-) mice [16, 17]. Salidroside induced autophagy and decreased apoptosis in HUVECs exposed to ox-LDL [18]. However, whether SIRT1 and FOXO1 mediate autophagy by salidroside remains unclear.

In this study, we investigated the underlying mechanism of the protective effects and autophagy of salidroside on ox-LDL-induced endothelial cell injury. We hypothesized that activation of SIRT1-FoxO1 pathway mediates autophagy induction, and reduced oxidative stress and endothelial cell injury by salidroside.

\section{Methods \\ Reagents and chemicals}

Human umbilical vein endothelial cells (HUVECs) were purchased from Cell Bank of Chinese Academy of Sciences (Shanghai, China). Salidroside (purity $>99 \%$, CAS: 43866), 3-Methyladenine (3-MA, CAS: M9281) and nicotinamide (NAM, CAS: 72340) were purchased from Sigma-Aldrich (Merck KGaA, Darmstadt, Germany). FOXO1 inhibitor AS1842856 (CAS: A15871) was purchased from AdooQ BioScience (Irvine, CA, USA). Ox-LDL was purchased from Beijing Solarbio Life Science Company (No. H7950; Beijing, China). Low glucose DMEM media and fetal bovine serum (FBS) were purchased from Invitrogen (Carlsbad, CA, USA). Cell counting kit-8 (CCK-8) kits was obtained from Beyotime Institute of Biotechnology (No. C0038; Shanghai, China). LDH assay kit (CAS: A020-3), MDA assay kit (CAS: A003-1), SOD assay kit (CAS: A001-1) and NADPH oxidase kit (CAS: A127) were purchased from Nanjing Jiancheng Bioengineering Institute (Nanjing, China). The primary antibodies against LC3 $\beta$ (CAS: sc-398,822), SIRT1 (CAS: sc-74,504) and FOXO1 (CAS: sc-374,427) were purchased from Santa Cruz Biotechnology (Santa Cruz, CA, USA).

\section{Cell culture and treatment}

HUVECs were cultured in DMEM (low glucose) supplemented with $10 \% \mathrm{FBS}$, and maintained in a humidified atmosphere containing $5 \% \mathrm{CO}_{2}$ at $37^{\circ} \mathrm{C}$. In vitro atherosclerosis model was established by incubation of HUVECs with different concentrations of ox-LDL $(0,10$, $20,50,100,150 \mu \mathrm{g} / \mathrm{mL}$ ) for $48 \mathrm{~h}$. Then $100 \mu \mathrm{g} / \mathrm{mL}$ of oxLDL was chosen as the propriate concentration and HUVECs were pretreated with salidroside at 20, 50, $100 \mu \mathrm{M}$ for $2 \mathrm{~h}$ and then exposed to ox-LDL $(100 \mu \mathrm{g} / \mathrm{mL})$ for $48 \mathrm{~h}$. The control group received $0.1 \%$ DMSO as vehicle. 


\section{Cell viability assay}

Cell viability was measured by cell counting kit-8 (CCK-8). HUVECs were seeded in 96-well plates at a density of $1 \times$ $10^{4}$ cells $/ \mathrm{mL}$, and then treated with different concentrations of ox-LDL $(0,10,20,50,100,150 \mu \mathrm{g} / \mathrm{mL})$, ox-LDL $(100 \mu \mathrm{g} / \mathrm{mL})$ and SAL $(20,50,100 \mu \mathrm{M})$ for $48 \mathrm{~h}$. After washing three times with PBS, cell were incubated with CCK- 8 solution and media (1:10 dilution) at $37^{\circ} \mathrm{C}$ for $1 \mathrm{~h}$, and then measured absorbance at $450 \mathrm{~nm}$ by a microplate reader.

\section{$\mathrm{LDH}$ release assay}

HUVECs were cultured in 96-well plates at a density of $1 \times 10^{4}$ cells $/ \mathrm{mL}$. HUVECs were pretreated with salidroside at $20,50,100 \mu \mathrm{M}$ for $2 \mathrm{~h}$ and then exposed to oxLDL $(100 \mu \mathrm{g} / \mathrm{mL})$ for $48 \mathrm{~h}$, then the LDH content in the media was assessed using a LDH activity kit.

\section{Determination of NADPH oxidase, MDA and SOD activities} HUVECs were plated $\left(1 \times 10^{5} / \mathrm{mL}\right)$ in 6 -well plates with DMEM medium containing $1 \%$ FBS. After $24 \mathrm{~h}$, HUVECs received different concentrations of ox-LDL, or ox-LDL $(100 \mu \mathrm{g} / \mathrm{mL})$ and salidroside $(20,50,100 \mu \mathrm{M})$, or ox-LDL $(100 \mu \mathrm{g} / \mathrm{mL})$, salidroside $(100 \mu \mathrm{M})$ and 3-MA, NAM or AS1842856, and cells were cultured for additional $48 \mathrm{~h}$. Then cells were harvested, lysed in PBS by ultrasonic pyrolysis and centrifuged at $3000 \times \mathrm{g}$ for 10 $\min$ at $4{ }^{\circ} \mathrm{C}$. A total of $100 \mu \mathrm{L}$ supernatant and were mixed with detection working fluid for NADPH oxidase, MDA or SOD at $37^{\circ} \mathrm{C}$ for $15 \mathrm{~min}$. Then the reaction mixture was centrifuged and transferred to 96-well plates, and a microplate reader was used to measure absorbance values at $340 \mathrm{~nm}$ (NADPH oxidase), at $532 \mathrm{~nm}$ (MDA), and at $520 \mathrm{~nm}$ (SOD).

\section{Immunofluorescence}

HUVECs $\left(2 \times 10^{4} / \mathrm{mL}\right)$ were seeded on circular coverslips in 6-well culture plates. In one experiment, cells were pretreated with salidroside at $20,50,100 \mu \mathrm{M}$ for 2 $\mathrm{h}$ and exposed to ox-LDL $(100 \mu \mathrm{g} / \mathrm{mL})$ for further $48 \mathrm{~h}$. In another experiment, cells were incubated with salidroside $(100 \mu \mathrm{M})$ and SIRT1 inhibitor nicotinamide (NAM, $100 \mathrm{nM}$ ) or FOXO1 inhibitor (AS1842856, 50 $\mathrm{nM})$ for $2 \mathrm{~h}$, an then incubated with ox-LDL $(100 \mu \mathrm{g} /$ $\mathrm{mL}$ ) for further $48 \mathrm{~h}$. After washing with PBS for 3 times, cultures were fixed $4 \%$ paraformaldehyde ( $\mathrm{pH}$ 7.4) for $20 \mathrm{~min}$, and blocked with $1 \%$ BSA and $0.1 \%$ TritonX-100 for $10 \mathrm{~min}$ at room temperature. HUVECs were incubated with goat polyclonal anti-LC3 $\beta$ antibody at $4^{\circ}$ $\mathrm{C}$ overnight, followed by PBS washing and incubation with fluorescein isothiocyanate (FITC) conjugated secondary antibody (IgG) for $1 \mathrm{~h}$ at $37^{\circ} \mathrm{C}$. After rinsing several times, the cells were incubated with DAPI (10 $\mathrm{mg} / \mathrm{mL}$ ) for $5 \mathrm{~min}$ at room temperature. Cultures were then mounted on glass slides and observed under a confocal microscope (Leica, Germany). The number of cells with punctate fluorescent LC3 was counted, and normalized to all DAPI fluorescent cells (For each group a minimum of 100 fluorescence-positive cells were counted), and presented as a percentage of cells with LC3 dots.

\section{Quantitative real-time PCR (qRT-PCR)}

Total RNA was isolated using TRIzol $^{\circ}$ reagent reversely transcribed into cDNA using Superscript II reverse transcriptase (Toyobo Life Science, Osaka, Japan). The qRT-PCR reaction system contained $2 \mu \mathrm{L}$ of cDNA sample solution, $10 \mu \mathrm{L}$ of SYBR-Green PCR master mix, $0.5 \mu \mathrm{L}$ of forward and reverse primers $(1 \mu \mathrm{M})$, and $7.5 \mu \mathrm{L}$ of $\mathrm{H}_{2} \mathrm{O}$. The primer sequences were as follows: SIRT1, forward 5'-GCC AGA GTC CAA GTT TAG AAG A3', reverse 5' -CCA TCA GTC CCA AAT CCA G-3'; FOXO1, forward 5'-GGC TGA GGG TTA GTG AGC AG-3' and reverse 5' -AAA GGG AGT TGG TGA AAG ACA-3' and GAPDH, forward 5'-CCT CAA GAT CAT CAG CAA TG-3' and reverse 5' -CCA TCC ACA GTC TTC TGG GT-3'. The amplification process was carried out as follows: denaturation at $95^{\circ} \mathrm{C}$ for $5 \mathrm{~min}$, followed by 40 cycles of denaturation at $95^{\circ} \mathrm{C}$ for $45 \mathrm{~s}$, annealing at $50{ }^{\circ} \mathrm{C}$ for $45 \mathrm{~s}$ and elongation at $72{ }^{\circ} \mathrm{C}$ for $45 \mathrm{~s}$, with the final extension step maintaining at $72^{\circ} \mathrm{C}$ for $10 \mathrm{~min}$. qRT-PCR was performed using an ABI Prism 7500 Fast Real-time PCR instrument (Applied Biosystems; Foster City, CA, USA), and analyzed using the $2-\Delta \Delta C$ t method. The mRNA levels of SIRT1 and FOXO1 were normalized to those of GAPDH to assess the significance of the differences between the groups.

\section{Western blotting}

Total protein was extracted from homogenate samples of HUVECs, the lysate was centrifuged to collect supernatant, and the protein concentration was determined by bicinchoninic acid assay. Total protein $(50 \mu \mathrm{g})$ was loaded in 10\% SDS-PAGE and then transferred to a nitrocellulose membrane. The membrane was then blocked with 5\% low fat milk and $0.05 \%$ Tween-20 in Tris buffered saline. Subsequently, the membrane was incubated with primary antibody (diluted 1:200) as mouse anti-SIRT1 and anti-FOXO1. After complete washing, the membrane was incubated with horseradish peroxidase-conjugated anti-mouse secondary antibody (1:250). The bands identified by the primary antibody were observed by a chemiluminescent detection system (ECL, Amersham Life Sciences, Buckinghamshire, UK). The optical density of the protein bands was analyzed using ImageJ software. The density values of SIRT1 and anti-FOXO1 were normalized to $\beta$-actin. 


\section{Statistical analysis}

Data are presented as means \pm standard deviation (SD), and analyzed by SPSS 19.0 statistical software. Comparisons between three or more groups were analyzed by analysis of variance (ANOVA), followed by the student Newman-Keuls (SNK) test. $P<0.05$ was considered as statistical significance.

\section{Results}

\section{Salidroside prevented the cytotoxic activity of ox-LDL in} HUVECS

In order to establish the in vitro atherosclerosis model, the HUVECs were exposed to various concentrations of ox-LDL $(10,20,50,100$ and $150 \mu \mathrm{g} / \mathrm{mL})$ for $48 \mathrm{~h}$. The cultured HUVECs displayed a reduction of cell viability dependent on various concentrations of ox-LDL, with significant reduction in cell viability at 50, 100 and $150 \mu \mathrm{g} / \mathrm{mL}$ (Fig. 1a). ox-LDL also induced oxidative injury in HUVECs, as evidenced by remarkably increased MDA and decreased SOD in all concentrations (Fig. 1b, c). Therefore, exposure to ox-LDL at $100 \mu \mathrm{g} / \mathrm{mL}$ was used in subsequent experiments. We then examined the effect of SAL on endothelial cell, and HUVECs were pretreated with SAL at 20, 50, $100 \mu \mathrm{M}$ for $2 \mathrm{~h}$ and exposed to ox-LDL $(100 \mu \mathrm{g} / \mathrm{mL})$ for further $48 \mathrm{~h}$. HUVEC morphology was observed under an inverted phase contrast microscope $(\times 10)$. ox-LDL treatment for $48 \mathrm{~h}$ demonstrate cellular fragmentations, vacuoles and debris, and these abnormal cellular morphologies were attenuated by SAL pretreatment, with more obvious improvement by SAL at $100 \mu \mathrm{M}$ compared with other two concentrations (Fig. 1d). CKK-8 assay showed that pretreatment with SAL significantly increased the cell viability at 20 and $50 \mu \mathrm{M}$ and suppressed the $\mathrm{LDH}$ release at 10, 20 and $50 \mu \mathrm{M}$ (Fig. 1e, f). However, SAL alone $(100 \mu \mathrm{M})$ had no significant effect on cell viability, and slightly increased LDH release in HUVECs without ox-LDL.

\section{Salidroside promoted autophagy and suppressed oxidative stress in HUVECs with ox-LDL}

To assess the regulation of SAL on autophagy in oxLDL treated HUVECs, we monitored the extent of autophagy by staining HUVECs with LC3 antibody by confocal microscopy. The FITC-positive green puncta mainly show autophagosomes, whereas the DAPIpositive blue puncta represent nucleus. The green puncta was merged with the blue puncta and appeared yellow images, which were indicators of autophagosomes. ox-LDL induced autophagy in HUVECs, as evidenced by slightly increased yellow fluorescence. SAL pretreatment further increased yellow fluorescence (Fig. 2a). Quantification analysis showed that SAL significantly increased the percentage of cells with LC3 dots (Fig. 2b). In order to investigate the effect of SAL on oxidative stress, we measured intracellular NADPH oxidase activity, MDA level and SOD activity. ox-LDL significantly increased NADPH oxidase activity (Fig. 2c) and MDA level (Fig. 2d), and decreased SOD activity (Fig. 2e) in HUVECs. However, these oxidative stress indicators could be significantly reversed by SAL in a concentration-dependent manner in ox-LDL-induced HUVECs $(P<0.05)$. Taken together, ox-LDL induced autophagy and oxidative stress in HUVECs, and SAL could further promote autophagy but exert an antioxidant effect on ox-LDL-induced HUVECs.

\section{Salidroside upregulated expressions of SIRT1 and FOXO1 in HUVECs with ox-LDL}

In order to explore underly mechanisms regulated by SAL, we measured mRNA and protein expressions of two genes which are associated with autophagy, SIRT1 and FOXO1. HUVECs were pretreated with SAL at 20, $50,100 \mu \mathrm{M}$ for $2 \mathrm{~h}$ and exposed to ox-LDL $(100 \mu \mathrm{g} / \mathrm{mL})$ for further $48 \mathrm{~h}$. qRT-PCR showed that compared with control cells, the mRNA expressions of SIRT1 and FOXO1 were significantly decreased after exposure to ox-LDL $(100 \mu \mathrm{g} / \mathrm{mL})$. Co-treatment with SAL significantly increased SIRT1 and FOXO1 mRNA expressions in a concentration-dependent manner (Fig. 3a, b). Western blotting also showed that SIRT1 and FOXO1 proteins were obviously decreased after exposure to ox-LDL, and were enhanced by SAL pretreatment (Fig. 3c, d). Furthermore, to investigated the mutual regulation between SIRT1 and FOXO1, HUVECs were treated with SAL $(100 \mu \mathrm{M})$, or with SAL $(100 \mu \mathrm{M})$ plus FOXO1 inhibitor (AS1842856, $100 \mathrm{nM}$ ), or with SAL $(100 \mu \mathrm{M})$ plus SIRT1 inhibitor nicotinamide (NAM, $200 \mathrm{nM}$ ). The results showed that increase in SIRT1 protein by SAL could be attenuated by AS1842856, and increase in FOXO1 protein by SAL could be obviously abolished by NAM (Fig. 3e, f). This indicates that there is inter-regulation between SIRT1 and FOXO1 in SAL-induced endothelial protection and autophagy promotion.

\section{SIRT1 and FOXO1 mediate endothelial injury, oxidative stress and autophagy by salidroside}

To investigate the role of SIRT1 and FOXO1 in autophagy induction by SAL, HUVECs were incubated with oxLDL $(100 \mu \mathrm{g} / \mathrm{mL})$ and SAL $(100 \mu \mathrm{M})$, in combination with NAM $(200 \mathrm{nM})$ or AAS1842856 $(100 \mathrm{nM})$. Compared with HUVECs with SAL alone, cotreatment with NAM $(200 \mathrm{nM})$ or AS1842856 (100 nM) obviously reduced fluorescence intensity and significantly decreased the percentages of cells with LC3 dots (Fig. 4a, b). We then investigated the role of autophagy, SIRT1 and FOXO1 in protection of endothelial injury and oxidative 

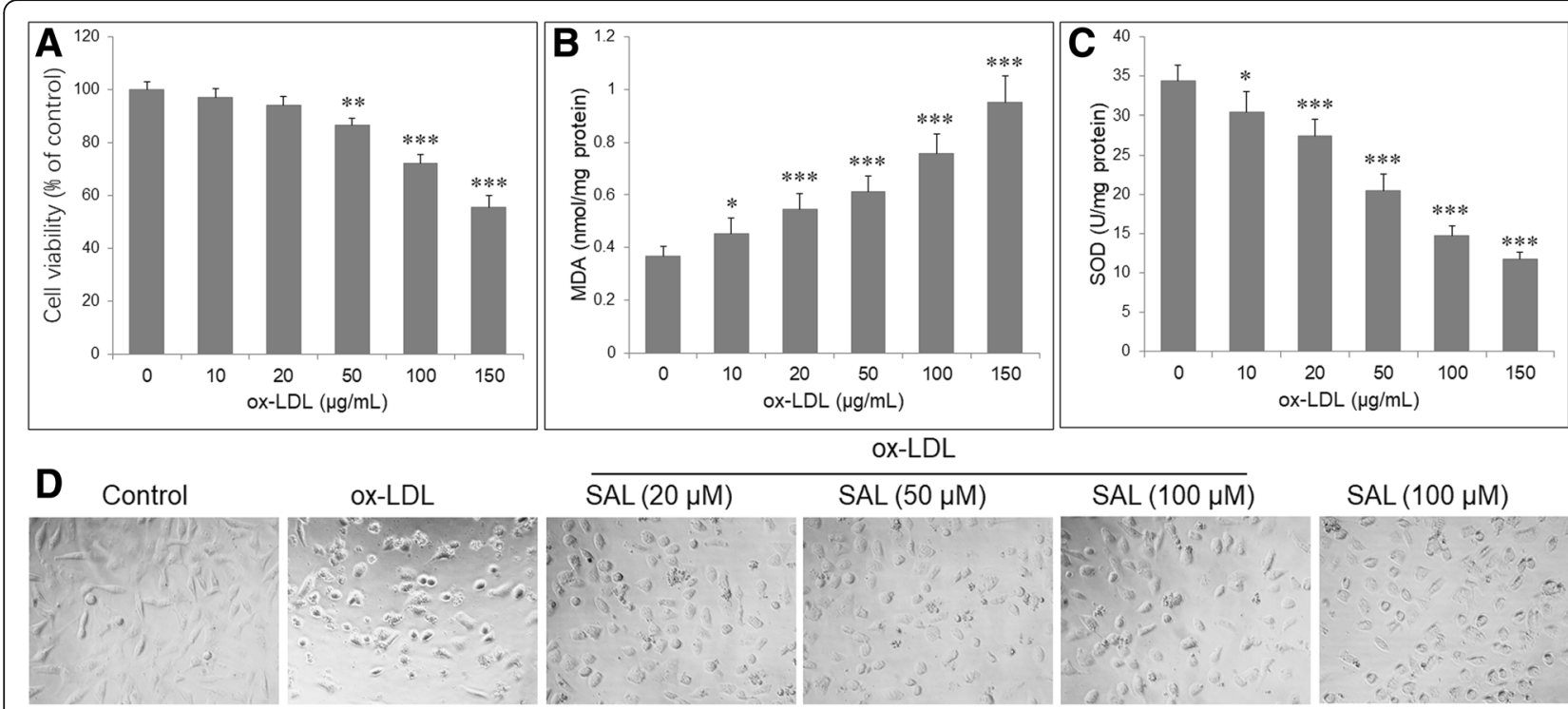

ox-LDL
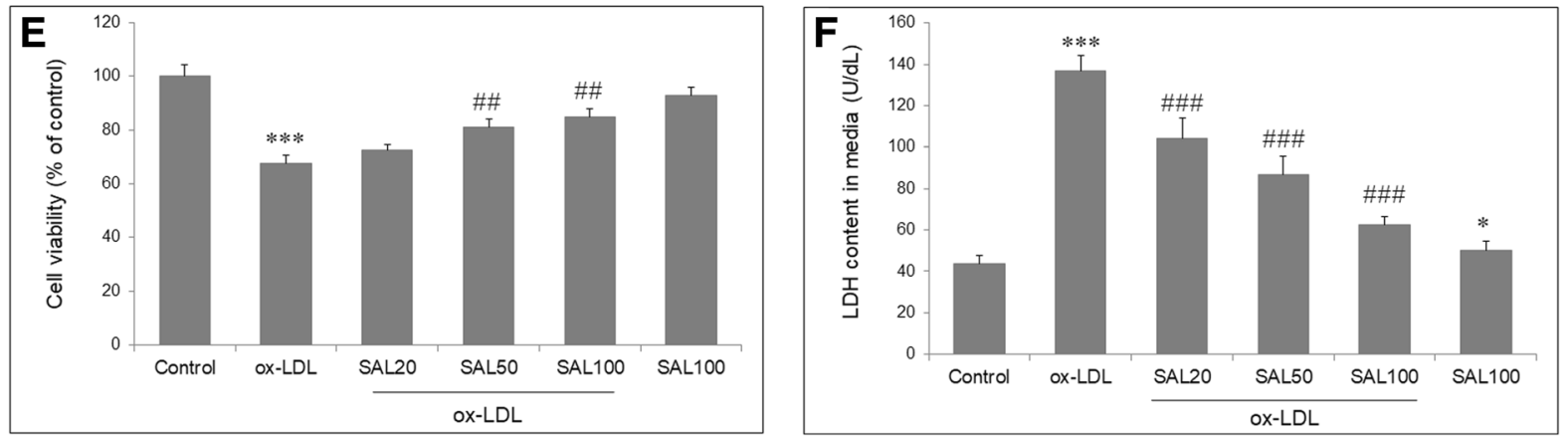

Fig. 1 Effect of salidroside on endothelial cell injury. HUVECs were exposed to various concentrations of ox-LDL (10, 20,50, 100 and 150 $\mu \mathrm{g} / \mathrm{mL})$ for $48 \mathrm{~h}$. a Cellular viability was detected by CKK-8 assay, which was normalized to control group. MDA level (b) and SOD enzymatic activity (c) in HUVECs were analyzed by chromometry using commercially available assay kits. d HUVECs were pretreated with SAL (20,50, 100 $\mu \mathrm{M})$ for $2 \mathrm{~h}$, and then incubated with ox-LDL $(100 \mu \mathrm{g} / \mathrm{mL})$ for $48 \mathrm{~h}$. HUVEC morphology was observed under an inverted phase contrast microscope $(\times 10)$. After $48 \mathrm{~h}$ of ox-LDL treatment, HUVECS demonstrate typical cellular fragmentations, vacuoles, and debris, which are obviously attenuated by SAL. SAL significantly attenuated the decrease cellular viability (e) and increase in LDH release (f) in HUVECs exposed to ox-LDL. Data are shown as mean $\pm \mathrm{SD}(n=6) .{ }^{*} P<0.05,{ }^{*} P<0.01,{ }^{* * *} P<0.001$ vs. control group; $\# P<0.01$, \#\#\#<0.001 vs. ox-LDL group

stress by SAL, by cotreatment with autophagy inhibitor 3Methyladenine (3-MA; $100 \mu \mathrm{M})$, NAM and AS1842856. Compared with HUVECs with ox-LDL and SAL, cells cotreated with 3-MA, NAM or AS1842856 significantly reduced cell viability (Fig. 4c), increased in MAD level (Fig. 4d), and decrease in SOD activity (Fig. 4e) in oxLDL-induced HUVECs.

\section{Discussion}

In this study, we investigated the protective effect of SAL on ox-LDL-induced endothelial injury. ox-LDL $(100 \mu \mathrm{g} / \mathrm{mL})$ exposure reduced cell viability and increased LDH release, which was attenuated by treatment with SAL (20, 50, $100 \mu \mathrm{M})$ for $48 \mathrm{~h}$. In HUVECs exposed to ox-LDL, SAL enhanced autophagy and suppressed oxidative stress. SAL attenuated the reduction in mRNA and protein expressions of SIRT1 and FOXO1 by ox-
LDL. SIRT1 inhibitor or FOXO1 inhibitor could abolish the suppression on oxidative stress and endothelial injury and enhancement on autophagy by SAL. Therefore, SAL protects HUVECs against ox-LDL-induced injury through upregulation of autophagy mediated by SIRT1FOXO1 axis.

In our experiment, HUVECs were incubated with $100 \mu \mathrm{g} / \mathrm{mL}$ of ox-LDL for $48 \mathrm{~h}$, and showed reduced viability. This indicates that ox-LDL induces oxidative injury in endothelial cells, as evidenced by increased MDA and decreased SOD. Thus, HUVECs with ROSderived oxidative injury could simulate endothelial dysfunction in atherosclerosis. Our data demonstrated that SAL exerted cytoprotective effects on endothelial injury, suppressed oxidative stress and enhanced autophagy. The increased cell viability may be related to increased percent of S phase cells and decreased apoptosis by SAL 


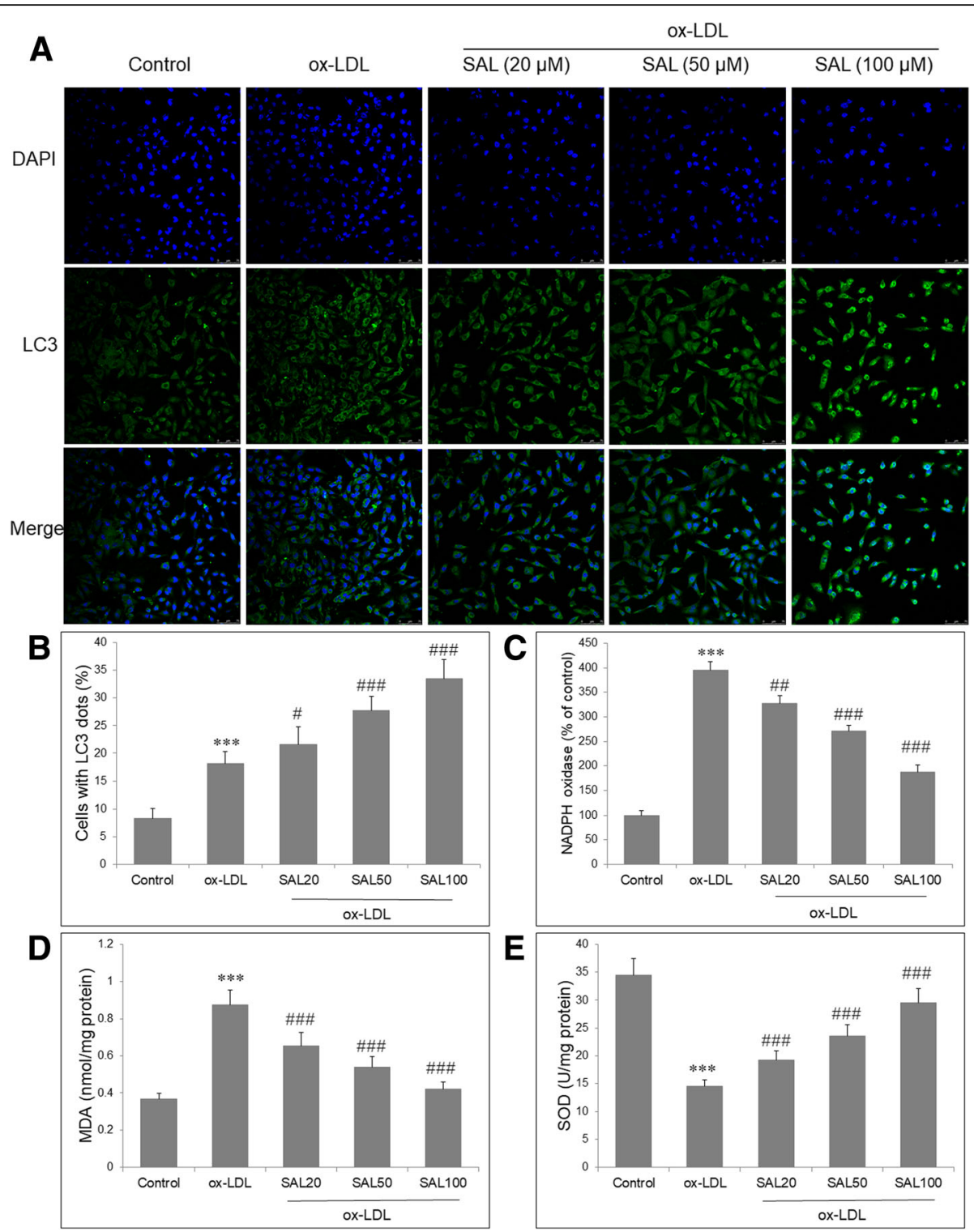

Fig. 2 Effect of salidroside on autophagy and oxidative stress in ox-LDL-induced HUVECs. a Fluorescence microscopy after immunofluorescence staining with an LC3 antibody, followed by FITC-labeled secondary antibody (green, original magnification $\times 100$ ). The nucleus was stained with DAPI (blue). $\mathbf{b}$ Quantification of fluorescence intensity of LC3, as presented by cells with LC3 dots. SAL decreases the NADPH oxidase activity (c), increases MDA level (d) and decreases SOD activity (e) in HUVECs exposed to ox-LDL $(100 \mu \mathrm{g} / \mathrm{mL})$. Data are shown as mean \pm SD $(n=6)$. ***P $<0.001$ vs. control group; $\# P<0.05$, \#\#P<0.01, \#\#P $<0.001$ vs. Ox-LDL group

in macrophages [16]. This is the third report about the effect of SAL on ox-LDL-induced cells. The previous reports showed that SAL suppressed foam cell formation and apoptosis in ox-LDL-induced THP1 cells [16], and prevented ox-LDL-treated endothelial cell senescence by increasing percentage of S phase cells [19]. Up to now, there is no report about the direct action of SAL on oxidized LDL. So SAL might interfere with the signal pathways induced by ox-LDL. For example, SAL prevented cytotoxicity in endothelial cell line EVC-304 and primary retinal endothelial cells induced by endogenous and exogenous hydrogen peroxide, respectively $[20,21]$. The underlying mechanisms are increased $\mathrm{Bcl} 2 / \mathrm{Bax}$ survival signaling pathway and activation of endogenous antioxidant enzymes, which is supported by our results that SAL attenuated cytotoxicity in HUVECs with decreased MDA and increased SOD.

Our study shows that SAL enhanced autophagy while suppressed oxidative stress in HUVECs induced with ox-LDL. The relationship between autophagy and oxidative stress remains controversial in atherosclerosis. Oxidative stress contributes to atherogenesis through 





and then incubated with ox-LDL $(100 \mu \mathrm{g} / \mathrm{mL})$ for $48 \mathrm{~h}$. qRT-PCR shows that the mRNA levels of SIRT1 (a) and FOXO1 (b) were significantly reduced by ox-LDL, but was increased by SAL in a concentration dependent manner. Western blotting was performed to determine protein

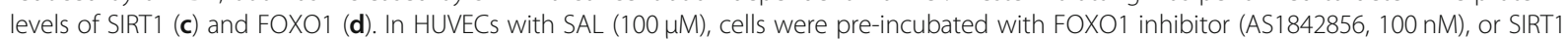
inhibitor nicotinamide (NAM, $200 \mathrm{nM}$ ). SAL significantly attenuates the decrease in SIRT1 (e) and FOXO1 (f) proteins in HUVECs exposed to ox-LDL. Data are shown as mean \pm SD $(n=6) .{ }^{* * *} P<0.001$ vs. control group; \#\#P<0.01, \#\#\#P<0.001 vs. ox-LDL group; $\$ \$ P<0.001$ vs. SAL $(100 \mu M)$ group

oxidation of LDL, which is cytotoxic to vascular cells. While autophagy is most likely a protective mechanism of cell in response to ox-LDL, thus allows atherosclerotic cells to survive [22]. This protective mechanism could be induced in atherosclerosis, as evidenced by activated autophagy in cultured HUVECs by ox-LDL and degradation of ox-LDL by autophagy activation [8]. Our data showed that ox-LDL increased autophagy in HUVECs, and SAL further enhanced autophagy in HUVECs exposed to ox-LDL. Cotreatment of HUVECs with an autophagy inhibitor 3-Methyladenine (3-MA) could abolish increased cell viability by SAL, and this suggests that autophagy induced by SAL is a protective mechanism against atherosclerosis. Our speculation is supported by another report, in which SAL increased autophagy and decreased apoptosis of HUVECs induced by $\mathrm{H}_{2} \mathrm{O}_{2}$ [18].

This study shows that SAL increased mRNA and protein expressions of SIRT1 in HUVECs exposed to oxLDL, and SIRT1 inhibition reduced autophagy, and reversed suppression on oxidative stress and endothelial injury. SIRT1 exerts protective roles in atherosclerosis through suppression of endothelial oxidative stress and foam cell formation ${ }^{10}$. SIRT1-mediated autophagy could protect HUVECs against Ox-LDL-induced injury by various agents, and our study adds SAL as another protective autophagy stimulator in HUVECs [23, 24]. Moreover, our study shows that SIRT1 has suppressive effect on oxidative stress, and this effect might be related to autophagy induction. The reason lies in the fact that reduced oxidative stress in HUVECs was also abolished by an autophagy inhibitor 3-MA, and one report that SIRT1-induced autophagy mediated suppressed oxidative stress by SAL in status epilepticus [25]. Thus, in oxLDL exposed HUVECs, SAL activates SIRT1, autophagy, and inhibits oxidative stress, thus protects against endothelial injury.

Our study also shows that FOXO1 mRNA and protein expressions was increased by SAL in HUVECs exposed to ox-LDL, which mediated enhanced autophagy, and suppressed oxidative stress and endothelial injury. FOXO1 regulates various genes involved in response to 

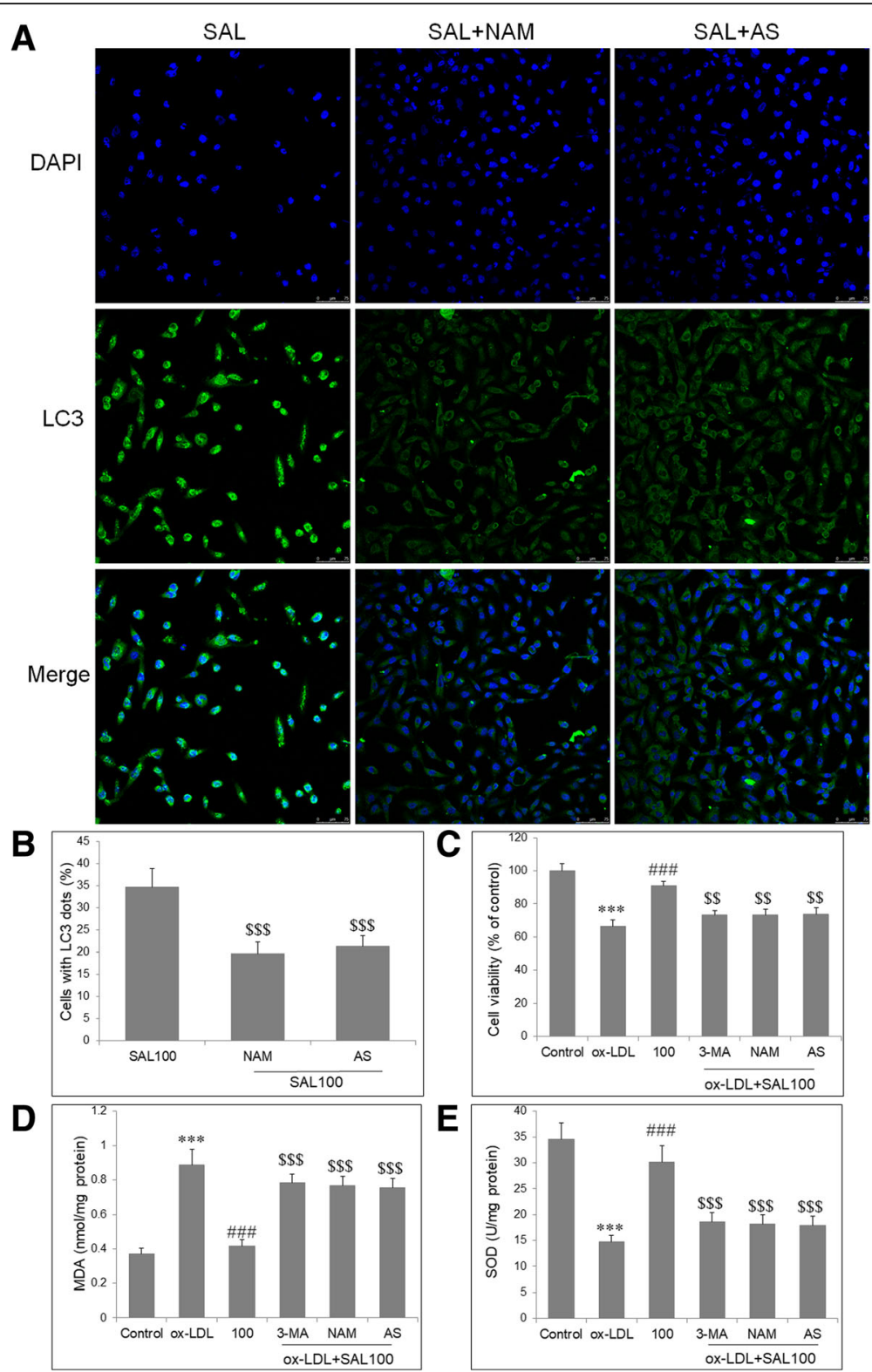

Fig. 4 SIRT1 and FOXO1 mediate the protective effects of SAL on OX-LDL-induced endothelial injury, oxidative stress and autophagy. a Autophagy of HUVECs stained with an LC3 antibody under fluorescence microscopy. In cells with ox-LDL and SAL (100 $\mu$ M), pretreatment with NAM (200 nM) or AS1842856 (100 nM) obviously reduced fluorescence intensity. b Quantification analysis shows that NAM and AS1842856 both significantly reduced the percentages of cells with LC3 dots. Autophagy inhibitor 3-Methyladenine (3-MA; $100 \mu$ M), NAM and AS1842856 all obviously abolish the protective effect of SAL on ox-LDL-induced endothelial injury (c), increase in MAD level (d), and decrease in SOD activity (e). Data are shown as mean $\pm S D(n=6) .{ }^{* * *} P<0.001$ vs. control group; \#\#\#P<0.001 vs. Ox-LDL group; $\$ P<0.01, \$ \$ P<0.001 \mathrm{vs.} \mathrm{SAL}$ $(100 \mu \mathrm{M})$ group

oxidative stress, and acts as a potent inhibitor of oxidative stress in various diseases with excessive oxidative stress [13]. Moreover, FOXO1 silencing in mouse endothelial cells prevented atherosclerosis, and indicates that FOXO1 is an atheroprotective molecule [14]. FOXO1 could protect cardiomyocytes against oxidative stressinduced apoptosis through inducing autophagy, which is consistent with our results that FOXO1 inhibitor abolished the autophagy induction and attenuated endothelial injury by SAL [26]. Furthermore, SIRT1 and FOXO1 interact with each other in response to oxidative stress. SIRT1 deacetylates FOXO1 and regulates its transcriptional activity, and FOXO1 increases SIRT1 transcription and expression through binding to SIRT1 promoter [27]. This mechanism is also confirmed by our study that in HUVECs with ox-LDL and SAL treatment, 
increase in SIRT1 protein was attenuated by FOXO1 inhibitor AS1842856, and increase in FOXO1 protein was also attenuated by SIRT1 inhibitor NAM. In fact, activation of SIRT1- FOXO1 signaling axis could inhibit oxidative stress in ischemia/reperfusion injury of heart [28], and enhance autophagy in protecting against apoptosis of osteoblast and reducing cellular senescence in skin exposed to UV irradiation [29, 30]. However, this SIRT1-FOXO1 regulatory loop can be disrupted by oxidative stress, thereby contributing to endothelial dysfunction such as aging and ischemia. The roles and mechanisms of SIRT1-FOXO1 regulatory loop in atherosclerosis remains unknown.

\section{Conclusion}

The present study shows that salidroside enhanced autophagy through upregulation modulation of SIRT1FOXO1 axis in HUVECs with ox-LDL exposure. The cross talk between autophagy and oxidative stress might contribute to the autophagic protection of HUVECs by salidroside. These findings suggest a novel role for salidroside in inducing the protective autophagy, which may be a potential therapeutic strategy in prevention of atherosclerosis.

\section{Abbreviations \\ 3-MA: 3-Methyladenine; CCK-8: Cell counting kit-8; FOXO1: Forkhead box 01; HUVECs: Human umbilical vein endothelial cells; LDH: Lactate dehydrogenase; LDL: Low density lipoprotein; MDA: Malondialdehyde; NAM: Nicotinamide; Ox-LDL: Oxidized low-density lipoprotein; GRT- PCR: Quantitative real-time polymerase chain reaction; SAL: Salidroside; SOD: Superoxide dismutase}

\section{Acknowledgements}

Not applicable.

\section{Author's contributions}

ZSZ designed supervised the study, and wrote the manuscript; JYL performed experiments, analyzed the data and revised the manuscript; XRZ performed experiments. All authors have read and agreed to publish this manuscript.

\section{Funding}

This study was supported by Key Specialty Construction Project of Pudong New District of Shanghai (Grant No: PWZzk2017-17). The funder provided financial support for our experiment.

\section{Availability of data and materials}

Data and materials are available upon request to the corresponding author.

\section{Ethics approval and consent to participate}

This study is based on cell line. We have obtained statement from local ethics committee ruled that no formal ethics approval was required in this particular case

\section{Consent for publication}

Not applicable.

\section{Competing interests}

The authors declare that they have no competing interests.
Received: 28 September 2018 Accepted: 21 May 2019

Published online: 30 May 2019

\section{References}

1. Watanabe T, Sato K, Itoh F, Noguchi Y, Fujimoto K, Koyama T, et al. Emerging roles for vasoactive peptides in diagnostic and therapeutic strategies against atherosclerotic cardiovascular diseases. Curr Protein Pept Sci. 2013;14:472-80.

2. Mannarino E, Pirro M. Endothelial injury and repair: a novel theory for atherosclerosis. Angiology. 2008;59:69S-72S.

3. Lubrano V, Balzan S. LOX-1 and ROS, inseparable factors in the process of endothelial damage. Free Radic Res. 2014;48:841-8.

4. Kattoor AJ, Pothineni NVK, Palagiri D, Mehta JL. Oxidative stress in atherosclerosis. Curr Atheroscler Rep. 2017;19:42.

5. Tabas I, Garcia-Cardena G, Owens GK. Recent insights into the cellular biology of atherosclerosis. J Cell Biol. 2015;209:13-22.

6. Choi AM, Ryter SW, Levine B. Autophagy in human health and disease. N Engl J Med. 2013;368:651-62.

7. Mialet-Perez J, Vindis C. Autophagy in health and disease: focus on the cardiovascular system. Essays Biochem. 2017;61:721-32.

8. Ding Z, Liu S, Wang X, Khaidakov M, Dai Y, Mehta JL. Oxidant stress in mitochondrial DNA damage, autophagy and inflammation in atherosclerosis. Sci Rep. 2013;3:1077.

9. Maiuri MC, Grassia G, Platt AM, Carnuccio R, Ialenti A, Maffia P. Macrophage autophagy in atherosclerosis. Mediat Inflamm. 2013;2013:14.

10. Kitada M, Ogura Y, Koya D. The protective role of Sirt1 in vascular tissue: its relationship to vascular aging and atherosclerosis. Aging (Albany NY). 2016:8:2290-307.

11. Yang $X$, Wei J, He Y, Jing T, Li Y, Xiao Y, et al. SIRT1 inhibition promotes atherosclerosis through impaired autophagy. Oncotarget. 2017;8:51447-61.

12. He W, Zhang A, Qi L, Na C, Jiang R, Fan Z, et al. FOXO1, a potential therapeutic target, regulates Autophagic flux, oxidative stress, mitochondrial dysfunction, and apoptosis in human cholangiocarcinoma QBC939 cells. Cell Physiol Biochem. 2018:45:1506-14.

13. Ponugoti B, Xu F, Zhang C, Tian C, Pacios S, Graves DT. FOXO1 promotes wound healing through the up-regulation of TGF- $\beta 1$ and prevention of oxidative stress. J Cell Biol. 2013:203:327-43.

14. Tsuchiya K, Tanaka J, Shuiqing Y, Welch CL, DePinho RA, Tabas I, et al. FoxOs integrate pleiotropic actions of insulin in vascular endothelium to protect mice from atherosclerosis. Cell Metab. 2012;15:372-81.

15. Wang J, Li JZ, Lu AX, Zhang KF, Li BJ. Anticancer effect of salidroside on A549 lung cancer cells through inhibition of oxidative stress and phospho-p38 expression. Oncol Lett. 2014;7:1159-64.

16. Ni J, Li Y, Li W, Guo R. Salidroside protects against foam cell formation and apoptosis, possibly via the MAPK and AKT signaling pathways. Lipids Health Dis. 2017;16:198

17. Xing SS, Yang XY, Zheng T, Li WJ, Wu D, Chi JY, et al. Salidroside improves endothelial function and alleviates atherosclerosis by activating a mitochondria-related AMPK/PI3K/Akt/eNOS pathway. Vasc Pharmacol. 2015:72:141-52.

18. Zheng XT, Wu ZH, Wei Y, Dai JJ, Yu GF, Yuan F, et al. Induction of autophagy by salidroside through the AMPK-mTOR pathway protects vascular endothelial cells from oxidative stress-induced apoptosis. Mol Cell Biochem. 2017;425:125-38

19. Sun L, Dou F, Chen J, Chi H, Xing S, Liu T, et al. Salidroside slows the progression of EA.hy 926 cell senescence by regulating the cell cycle in an atherosclerosis model. Mol Med Rep. 2018;17:257-63.

20. X Z, Jin L, Shen N, Xu B, Zhang W, Zhu H, Luo Z. Salidroside inhibits endogenous hydrogen peroxide induced cytotoxicity of endothelial cells. Biol Pharm Bull. 2013;36:1773-8.

21. Shi K, Wang X, Zhu J, Cao G, Zhang K, Su Z. Salidroside protects retinal endothelial cells against hydrogen peroxide-induced injury via modulating oxidative status and apoptosis. Biosci Biotechnol Biochem. 2015;79:1406-13.

22. Perrotta I, Aquila S. The role of oxidative stress and autophagy in atherosclerosis. Oxidative Med Cell Longev. 2015;2015:130315.

23. Jin X, Chen M, Yi L, Chang H, Zhang T, Wang L, et al. Delphinidin-3glucoside protects human umbilical vein endothelial cells against oxidized low-density lipoprotein-induced injury by autophagy upregulation via the AMPK/RIRT1 signaling pathway. Mol Nutr Food Res. 2014;58:1941-51. 
24. Jiang Q, Hao R, Wang W, Gao H, Wang C. SIRT1/Atg5/autophagy are involved in the antiatherosclerosis effects of ursolic acid. Mol Cell Biochem. 2016;420:171-84

25. Si PP, Zhen JL, Cai YL, Wang WJ, Wang WP. Salidroside protects against kainic acid-induced status epilepticus via suppressing oxidative stress. Neurosci Lett. 2016;618:19-24.

26. Ning Y, Li Z, Qiu Z. FOXO1 silence aggravates oxidative stress-promoted apoptosis in cardiomyocytes by reducing autophagy. J Toxicol Sci. 2015;40:637-45

27. Xiong S, Salazar G, Patrushev N, Alexander RW. FoxO1 mediates an autofeedback loop regulating SIRT1 expression. J Biol Chem. 2011;286:5289-99.

28. Guan XH, Liu XH, Hong X, Zhao N, Xiao YF, Wang LF, et al. CD38 deficiency protects the heart from ischemia/reperfusion injury through activating SIRT1/FOXOs-mediated Antioxidative stress pathway. Oxidative Med Cell Longev. 2016;2016:7410257.

29. Gu X, Han D, Chen W, Zhang L, Lin Q, Gao J, et al. SIRT1-mediated FoxOs pathways protect against apoptosis by promoting autophagy in osteoblast-like MC3T3-E1 cells exposed to sodium fluoride. Oncotarget. 2016;7:65218-30.

30. Lim CJ, Lee YM, Kang SG, Lim HW, Shin KO, Jeong SK, et al. Aquatide activation of SIRT1 reduces cellular senescence through a SIRT1-FOXO1autophagy Axis. Biomol Ther (Seoul). 2017;25:511-8.

\section{Publisher's Note}

Springer Nature remains neutral with regard to jurisdictional claims in published maps and institutional affiliations.

Ready to submit your research? Choose BMC and benefit from:

- fast, convenient online submission

- thorough peer review by experienced researchers in your field

- rapid publication on acceptance

- support for research data, including large and complex data types

- gold Open Access which fosters wider collaboration and increased citations

- maximum visibility for your research: over $100 \mathrm{M}$ website views per year

At BMC, research is always in progress.

Learn more biomedcentral.com/submissions 